\title{
Configuration Optimization of Hypersonic Vehicles under Transitional Flow Conditions
}

\author{
Quanhua Sun ${ }^{1}$, Jing Fan $^{2}$, Jianzheng Jiang ${ }^{3}$, Kai Cui ${ }^{4}$ \\ LHD, Institute of Mechanics, Chinese Academy of Sciences, Beijing 100190
}

\begin{abstract}
The rarefied gas effects on several configurations are investigated under hypersonic flow conditions using the direct simulation Mont Carlo method. It is found that the Knudsen number, the Mach number, and the angle of attack all play a mixed role in the aerodynamics of a flat plate. The ratio of lift to drag decreases as the Knudsen number increases. Studies on 3D delta wings show that the ratio of lift to drag could be increased by decreasing the wing thickness and/or by increasing the wing span. It is also found that the waveriders could produce larger ratio of lift to drag as compared with the delta wing having the same length, wing span, and cross section area.
\end{abstract}

\section{Nomenclature}

$A \quad=$ projected wing area

$C_{A} \quad=$ axial or parallel force coefficient

$C_{D} \quad=$ drag coefficient

$C_{L} \quad=$ lift coefficient

$C_{N} \quad=$ normal force coefficient

$C_{p} \quad=$ pressure coefficient

$F_{X} \quad=$ axial or parallel force

$F_{N} \quad=$ normal force

$F_{X} \quad=\quad X$-component force

$F z \quad=\quad Z$-component force

$H \quad=$ wing thickness

Kn $\quad=$ Knudsen number

$L=$ plate or wing length

$\mathrm{Ma}=$ Mach number

$p \quad=$ pressure

$U \quad=$ velocity

$W \quad=$ wing span

$\rho=$ mass density

$\infty \quad=$ free stream condition

\section{Introduction}

$\mathrm{T}$

HERE are many vehicles flying in the atmosphere and having various shapes. Supersonic air planes usually have sharp leading edges to avoid bow shocks, whereas space planes use blunt bodies to protect strong thermal loads during the re-entry. Typically, these vehicles are optimized for their aerodynamics under continuum flow conditions since the aerodynamics is unimportant when there is no air or gas. However, it is well known that the rarefied gas effects during low-density flights will affect the performance of the vehicles. Then the aerodynamics of hypersonic vehicles under rarefied conditions has to be investigated and the configuration of vehicles might to be optimized to achieve better performance.

\footnotetext{
${ }^{1}$ Research Professor, Institute of Mechanics, Chinese Academy of Sciences, Beijing 100190, Senior Member AIAA.

${ }^{2}$ Research Professor, Institute of Mechanics, Chinese Academy of Sciences, Beijing 100190.

${ }^{3}$ Assistant Research Professor, Institute of Mechanics, Chinese Academy of Sciences, Beijing 100190.

${ }^{4}$ Associate Research Professor, Institute of Mechanics, Chinese Academy of Sciences, Beijing 100190.
} 
Related studies can be dated back to the sixties of the last century [1]. Early investigation usually combined the wind tunnel measurements, flight data, and simplified theoretic analysis. These ground studies can hardly explain the flight data because the wind tunnel could not reproduce the real flight conditions and theories were oversimplified. After Bird designed and developed the direct simulation Monte Carlo (DSMC) method [2], the DSMC method has been routinely used to analysis the hypersonic flows under rarefied flow conditions. The investigated configurations include waverider, lift body, space shuttle, delta wing, flat plate, cone, wedge, sphere, and cylinder. The DSMC simulations, however, are limited to very few cases for a specific configuration due to relatively large computational cost of the simulations. Thus related systematic studies can be hardly found in the literature.

In order to illustrate the rarefied gas effects on the aerodynamic performance of hypersonic bodies, we include typical results collected from the literature in figure 1 where the Knudsen number is estimated based on the mean free path of the undisturbed flow and the vehicle length. Although the data are based on different flow Mach number and angle of attack, two conclusions can be easily drawn. First, the ratio of lift to drag of hypersonic vehicles decreases when the flow Knudsen number increases. Second, the ratio of lift to drag is vehicle-dependent for the wide range of the Knudsen number. Therefore it is very important to optimize the vehicle's configuration under rarefied conditions since the aerodynamic performance of vehicles is poor for large Knudsen number flows.

In this paper, we intend to study the aerodynamics (especially the ratio of lift to drag) of model hypersonic vehicles under transitional flow conditions and provide preliminary optimization strategy. The optimization process is based on some simple configurations because it is too luxury to perform a vast number of tests as in CFD. The rest of the paper is organized as follows. Section II describes the employed simulation approach. Section III discusses the aerodynamics of a flat plate at a wide range of flow conditions, which will show the detailed rarefied gas effects for this simple geometry. Section IV analyzes the aerodynamics of several 3-dimensional configurations with an aim to provide a base for further optimization. Finally, some concluding remarks are given in Section V.

\section{Numerical Approach}

The direct simulation Monte Carlo method is employed for this study. The DSMC method simulates a gas flow through the computations of the motion and collisions of a large number of representative particles. The primary approximation (major difference against molecular dynamics methods) is to uncouple the molecular motions and intermolecular collisions over small time intervals, where particle motions are modeled deterministically and the collisions are treated statistically. In this study, the variable hard sphere (VHS) molecular model is employed to treat particle collisions, and the Larsen-Borgnakke phenomenal model is used to handle the exchange between translational energy and molecular internal energy.

In the simulation, the computational domain is divided into a net of small cells where the cell size is typically smaller than the local mean free path. When the cell size is larger, the selection of colliding particles is restricted to be within a short distance. The air is modeled as a single species where the rotational and vibrational energy is included. The thermal accommodation coefficient of the vehicle surface is assumed to be 1.0 and the surface temperature is 300 Kelvin. A typical simulation employs about 50,000,000 particles and it runs on multiple processors. In order to have good parallel efficiency, a load balance strategy is employed. The number of simulated particles in each processor is nearly the same. In addition, the cut of the computational domain is biased to be along the streamlines. A sample computational domain having multiple divisions is shown in figure 2 where the free stream comes from the left.

\section{Hypersonic Aerodynamics of Flat Plate from Near Continuum to Rarefied}

As figure 1 shows that the Knudsen number has strong effects on the aerodynamics of hypersonic vehicles, we investigate the hypersonic flow over a flat plate to show effects of various factors such as Knudsen number, angle of attack, and Mach number. The length of the plate is fixed at $1 \mathrm{~m}$.

It is well known that a shock wave will appear in a supersonic flow condition and the thickness of a shock wave is about several mean free paths. Then when the flow Knudsen number increases, the shock wave will become thicker and thicker. Figure 3 shows the pressure field (nondimensionalized using the free stream pressure) for the Knudsen number ranges from 0.002 to 2.0 when the free stream Mach number is 15 . When the Knudsen number is 0.002 , the oblique shock wave is clearly identified. The largest pressure is observed near the leading edge and then decreases along the plate. However, when there is non-zero angle of attack, the pressure in the leeward surface is below the free stream value. Thus the contribution to the total force from this side is negligible. In fact, there is an expansion fan in the leeward side (angle of attack is $10^{\circ}$ ). When the Knudsen number is increased to 0.02 , a clear shock wave is not observed because the thick shock wave has mixed with the boundary layer. The pressure behind the shock wave is larger than the corresponding pressure when $\mathrm{Kn}=0.002$. In addition, the range of the affected area 
is increased (the computational domain is also increased). Notice that the bow shock wave is also observed ahead of the expansion fan in the leeward side for the case when the angle of attack is $10^{\circ}$. When the Knudsen number becomes even larger, a shock wave cannot be identified and the affected area is increased. For instance, when $\mathrm{Kn}=2.0$, there is no much pressure difference between the leading edge and trailing edge. The leeward pressure for the non-zero angle of attack cases is also increased. The effects of the Knudsen number can also be observed on the streamlines. The streamlines got deflected for low Knudsen number cases whereas there is no obvious change for large Knudsen number cases. All these indicate that the flow structure changes with the Knudsen number.

The force exerted on the plate is then calculated using the surface pressure and shear stress. For the comparison purpose, the force is nondimensionalized as drag coefficient and lift coefficient that are calculated from the parallel (or axial) force coefficient and the normal force coefficient.

$$
\begin{aligned}
& C_{D}=\frac{F_{X}}{0.5 \rho_{\infty} U_{\infty}^{2} L} \\
& C_{L}=\frac{F_{Z}}{0.5 \rho_{\infty} U_{\infty}^{2} L} \\
& C_{A}=\frac{F_{A}}{0.5 \rho_{\infty} U_{\infty}^{2} L} \\
& C_{N}=\frac{F_{N}}{0.5 \rho_{\infty} U_{\infty}^{2} L}
\end{aligned}
$$

Figure 4 shows the aerodynamics of the hypersonic flat plate. The data are all calculated using the DSMC method except those free molecular results added at a position of $\mathrm{Kn}=80$ and others specified. Clearly, all the coefficients increase with the angle of attack for the range being investigated. The Knudsen number effects on these coefficients are quite complicated. When the angle of attack is zero, the parallel force coefficient (or averaged friction coefficient) increases with the Knudsen number and reaches to the maximum when the Knudsen number is about 0.1 , then decreases gradually and approaches to the free molecular value. When the angle of attack increases, the parallel force coefficient also increases with the Knudsen number, but reaches to its maximum at a larger Knudsen number or even reaches to the free molecular value without having a local maximum. It is also noticed that the effect of the angle of attack on the parallel force coefficient increases with the Knudsen number. For instance, when $\mathrm{Kn}=0.002$, the coefficient when the angle of attack is $30^{\circ}$ is about twice of that when the angle of attack is zero. However when $\mathrm{Kn}=10$, the difference is increased to more than four times. In addition, the parallel force coefficient at $\mathrm{Kn}=10$ is very close to the free molecular results.

The normal force coefficient behaves different from the parallel force coefficient regarding to the Knudsen number. When the Knudsen number is less than 0.001, the normal force coefficient is nearly independent on the Knudsen number (not shown), which is not the case for the parallel force coefficient. When the Knudsen number is larger than 0.001, the normal force coefficient increases with the Knudsen number and reaches to a maximum when the Knudsen number is around 0.1. Specifically, the maximum is reached at $\mathrm{Kn}=0.2$ when the angle of attack is larger than $5^{\circ}$. After the parallel force coefficient reaches its maximum, it gradually decreases to reach the free molecular results as the Knudsen number keeps increasing. The variation of the normal force coefficient, however, is much less than the variation of the parallel force coefficient with the change of the Knudsen number.

The behavior of the drag coefficient is similar to the parallel force coefficient. The main difference is that the drag coefficient at larger angle of attack is much larger than the corresponding parallel force coefficient because of the contribution from the normal force coefficient. It is noticed that the drag coefficient approaches to the free molecular data much faster than the parallel force coefficient does. The Knudsen number effect on the lift coefficient depends strongly on the angle of attack. When the angle of attack is small (less then $20^{\circ}$ ), the lift coefficient increases with the Knudsen number up to $\mathrm{Kn}=0.1$ and then decreases to free molecular results. However, when the angle of attack is larger than $20^{\circ}$, the lift coefficient decreases slowly with the Knudsen number and then 
drops quickly after the Knudsen number is larger than 0.1. The free molecular results, however, are still much smaller than the values when $\mathrm{Kn}=10$.

The ratio of lift to drag is plotted in figure 4(e). Clearly, the Knudsen number has strong effect on the value. For instance, the ratio of lift to drag is close to 1.0 when $\mathrm{Kn}=0.02$, but it is less than half when $\mathrm{Kn}=1.0$ for the case when the angle of attack is larger than $10^{\circ}$. It is also noticed than the angle of attack has obvious effect on the ratio of lift to drag at small Knudsen number and the effect decreases when the Knudsen number increases. Figure 4(f) re-plots the ratio of lift to drag as a function of the angle of attack. This plot shows that the ratio of lift to drag reaches the maximum at about $15^{\circ}$ when $\mathrm{Kn}=0.002$. Then when the Knudsen number increases, the ratio of lift to drag reaches to the maximum at a larger angle of attack $\left(25^{\circ}\right.$ when $\left.\mathrm{Kn}=0.05\right)$. But at some point, the ratio of lift to drag reaches to its maximum at a smaller angle of attack, which is consistent with the free molecular results that the maximum is achieved at the angle of attack about $5^{\circ}$. Therefore, the Knudsen number affects the angle of attack when the ratio of lift to drag is the maximum. This is also true for the continuum flows and the corresponding Navier-Stokes results are plotted in figure $4(\mathrm{~g})$.

Figure 4(h) shows the Mach number effects on the ratio of lift to drag. It seems that higher speed flows have larger ratio of lift to drag when the angle of attack is $5^{\circ}$. However, at larger angle of attack, lower hypersonic flows have larger ratio of lift to drag, which is the opposite. It is also noticed that the Mach number effect on the ratio of lift to drag decreases when the Mach number is larger than 15 and the angle of attack is larger than $10^{\circ}$.

In general, when the Knudsen number increases, the drag coefficient increases and the lift coefficient decreases, thus makes the ratio of lift to drag decreases when the Knudsen number increases. Of course, the flight angle of attack and the free stream Mach number also play their roles in the aerodynamics of the hypersonic flat plate.

\section{Configuration Optimization}

Figure 4 has shown that the Knudsen number has strong effects on the aerodynamics on a hypersonic body. Especially the ratio of lift to drag is quite small when the flow is rarefied. In this section, we intend to investigate the aerodynamics of a 3D body where its geometry has several changes when the flow is rarefied.

\section{A. Delta Wings}

Delta wing has often been studied in the literature because of its simple geometry [7,10,11]. However, the size of the wing is difference from case to case. In this study, we choose the length of the delta wing is $1 \mathrm{~m}$ and the configuration is shown in figure 5. Figure 5(a) is the base configuration where the span is about $0.3 \mathrm{~m}$ and the wing thickness is about $0.06 \mathrm{~m}$. Figure $5(\mathrm{~b})$ is a variation where the location of the maximum thickness is shifted to $\mathrm{X}=0.8 \mathrm{~m}$. Figure $5(\mathrm{c})$ is the case when the wing height is reduced to half, and figure $5(\mathrm{~d})$ is another case where the wing height is half of the base case and the wing span is doubled.

Figure 6 shows the aerodynamics of the delta wings when the free stream Mach number is 25 and the Knudsen number is 0.015 . It seems that the drag coefficient has slight increase when the flight angle of attack increases from $0^{\circ}$ to $5^{\circ}$, then increases quickly with the angle of attack. Among the four configurations, the base configuration has the largest drag coefficient. The drag coefficient of configuration (b) is nearly the same as this of the base configuration at zero angle of attack, and then becomes smaller than the base configuration when the angle of attack increases. Configuration (c) has a smaller drag coefficient because its wing height is only half of the base configuration. However, it is surprised to see that configuration (d) has larger drag coefficient than configuration (c) although the former is relative thinner than the latter. Nevertheless, the difference of the drag coefficient is less than $15 \%$ among all these configurations.

The configuration, however, has obvious effects on the lift coefficient. It is found that configuration (b) has smallest lift coefficient. Configuration (a) and (c) have similar lift coefficient for all the angles of attack under considered, whereas configuration (d) has largest lift coefficient. Notice that all configurations have a small lift at zero angle of attack. The ratio of lift to drag is then calculated based on the lift and drag as shown in figure 6(c). It turns out that configuration (d) has largest value because of its lift; configuration (c) is the second because of its drag advantage; configuration (a) is the third, whereas configuration (b) is the worst due to its smallest lift. Clearly, the ratio of lift to drag of a hypersonic vehicle could be increased if its wing height gets decreased or/and its wing span increases.

\section{B. Waveriders}

Waveriders are preferred for hypersonic flights because they have relatively large ratio of lift to drag under continuum flow conditions. For this reason, we compare the aerodynamics of the delta wing with waveriders for rarefied gas flows. Two types of waveriders are considered: caret waverider and cone-derived waverider. Both 
waveriders have the same length and wing span as the delta wing as shown in figure 7 . The inclined angle of the waverider wings is the same at about $42^{\circ}$ and the cross section area is also the same as the base configuration of the delta wing.

Figure 8 shows the comparison of the aerodynamics of the delta wing and the waveriders when the free stream Mach number is 25 and the Knudsen is 0.015 . It seems that the delta wing has smaller drag coefficient, but its lift is even smaller than those of waveriders. Thus the waveriders have larger ratio of lift to drag than the delta wing. The difference of the aerodynamics between the caret waverider and cone-derived waverider, however, is rather small except that the cone-derived waverider has slightly larger ratio of lift to drag. The larger lift of waveriders comes from the fact that the waveriders have larger pressure on the facing surface as shown in figure 9.

The Knudsen number effects on the aerodynamics of waveriders are also investigated. Figure 10 shows the aerodynamics of the caret waverider when the free stream Mach number is 25. Clearly, the drag coefficient increases with the Knudsen number. However, when the Knudsen number increases from 0.003 to 0.15 , the lift coefficient is increased at small angle of attack but is decreased when the angle of attack is larger than $15^{\circ}$. In addition, the lift coefficient decreases when the Knudsen number increases from 0.015 to 0.1 . The ratio of lift to drag, on the other hand, is monotonically decreased when the Knudsen number increases.

\section{Conclusion}

The rarefied gas effects on the aerodynamics of hypersonic bodies were studied. The effects are somehow complicated because the flight angle of attack, the free stream Mach number, and the Knudsen number all play a role on the aerodynamics. In general, the ratio of lift to drag of a hypersonic body is decreased when the Knudsen number increases. When the angle of attack is small, there is a maximum for the drag coefficient at one Knudsen number and a maximum for the lift coefficient at another Knudsen number for the flat plate. Increasing the flight Mach number could increase the ratio of lift to drag at small angle of attack, but will decreases the ratio of lift to drag at large angle of attack.

When the Knudsen number is about 0.015 , the aerodynamics of delta wing type body was investigated. It is found that the ratio of lift to drag could be increased by decreasing the wing height and/or increasing the wing span. Another strategy for increasing the ratio of lift to drag is to use waveriders because of their larger lift.

The aerodynamics of hypersonic vehicles has been shown to be relatively poor under rarefied conditions. In addition, factors such as flight angle of attack and Mach number have mixed effects on the aerodynamics. Hence, careful optimization for a specific type of configuration is not only useful, but also important.

\section{Acknowledgments}

The authors would like to thank Prof. Zuowu Li for helpful discussions. This work is supported by the National Natural Science Foundation of China (Grants No. 50836007, No. 90816012, and No. 10621202).

\section{References}

${ }^{1}$ Potter, J. L. “The Transitional Rarefied Flow Regime," Rarefied Gas Dynamics, Vol. 2, Academic Press, 1967.

${ }^{2}$ Bird, G. A. Molecular gas dynamics, Clarendon Press, 1976.

${ }^{3}$ Tai, T. C., "Direct Simulation of Low-Density Flow over Airflows," AIAA 90-1539.

${ }^{4}$ Sunada, S., Sakaguchi, A., and Jawachi, K., "Airfoil Section Characteristics at a Low Reynolds Number," Journal of Fluids Engineering, Vol. 119, 1997, pp. 129-135.

${ }^{5}$ Anderson, J. D., Ferguson, F., and Lewis, M. J., "Hypersonic Wave-riders for High Altitude Applications," AIAA 91-0530.

${ }^{6}$ Cui, K., Zhao, D., and Yang, G., "Waverider Configurations Derived from General Conical Flowfields," Acta Mech Sin, Vol. 23, 2007, pp. 247-255.

${ }^{7}$ Rault, D. F. G., “Aerodynamic Characteristics of a Hypersonic Viscous Optimized Waverider at High Altitudes," Journal of Spacecraft and Rockets, Vol. 31(5), 1994, pp. 719-727.

${ }^{8}$ Potter, J. L., and Rockaway, J. K., "Aerodynamic Optimization for Hypersonic Flight at Very High Altitudes," Rarefied Gas Dynamics: Space Science and Engineering, 1993, 296-307.

${ }^{9}$ Kautz, F. A., and Baron, J. R., "Direct Simulation of Waveriders in Hypersonic Rarefied Flow," AIAA 91-1317.

${ }^{10}$ Celenligil, M. C., and Moss, J. N., "Direct Simulation of Hypersonic Rarefied Flow about a Delta Wing," AIAA 90-0143.

${ }^{11}$ Celenligil, M. C., and Moss, J. N., "Hypersonic Rarefied Flow about a Delta Wing - Direct Simulation and Comparison with Experiment," AIAA Journal, 30(8), 1992, pp. 2017-2023.

${ }^{12}$ Dogra, V. K., and Moss, J. N., "Hypersonic Rarefied Flow about Plates at Incidence," AIAA 89-1712.

${ }^{13}$ Rault, D. F. G., "Aerodynamics of the Shuttle Orbiter at High Altitudes," Journal of Spacecraft and Rockets, Vol. 31(6), 1994, pp. 944-952.

${ }^{14}$ Celenligil, M. C., Moss, J. N., and Blanchard, R. C., "Three-Dimension Rarefied Flow Simulations for the Aeroassist Flight Experiment Vehicle," AIAA Journal, Vol. 29(1), pp. 52-57. 
${ }^{15}$ Bird, G. A., Molecular gas dynamics and the direct simulation of gas flows, Clarendon Press, 1994.

${ }^{16}$ Becker, M., Bachour, F., Webber, E., and Erdtel, D., "Forces and Heat Transfer on Blunted Cones in the Transition Regime," Proceedings of the 9th International Symposium on Rarefied Gas Dynamics, 1974, D9.

${ }^{17}$ Rault, D. F. G., Wilmoth, R. G., and Bird, G. A. "An Efficient DSMC Algorithm Applied to a Delta Wing,” AIAA 91-1316.

${ }^{18}$ Riabov, V. V., "Comparative Similarity Analysis of Hypersonic Rarefied Gas Flows Near Simple-Shape Bodies," AIAA 972226.

${ }^{19}$ Potter, J. L., "Transitional, Hypervelocity Aerodynamic Simulation and Scaling," Progress in Astronautics and Aeronautics: Thermo-physical Aspects of Re-Entry Flows, Vol. 103, 1986, pp. 79-96.

${ }^{20}$ Potter, J. L., "Procedure for Estimating Aerodynamics of Three-Dimensional Bodies in Transitional Flow," Progress in Astronautics and Aeronautics, Vol. 118. 1989, pp. 484-492.

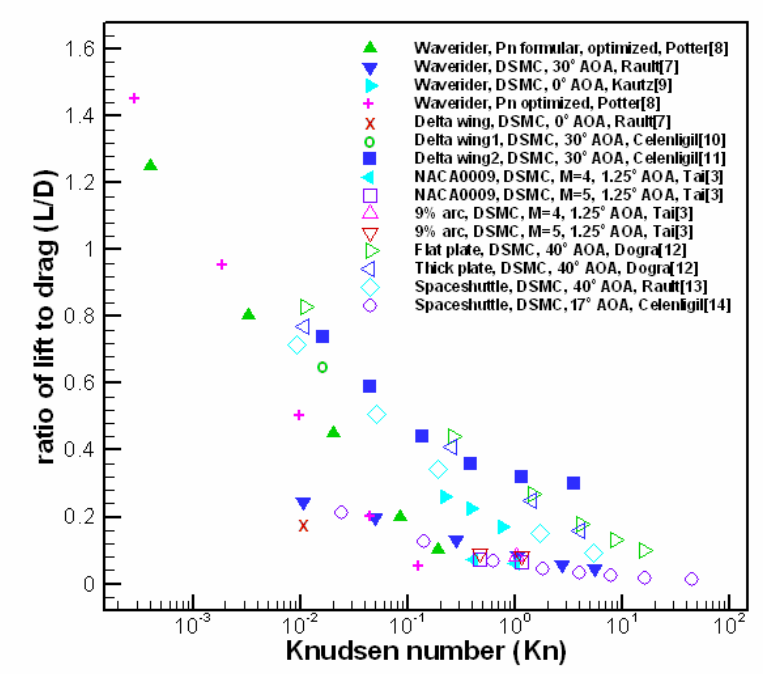

Figure 1. Ratio of lift to drag of hypersonic vehicles as a function of the Knudsen number.

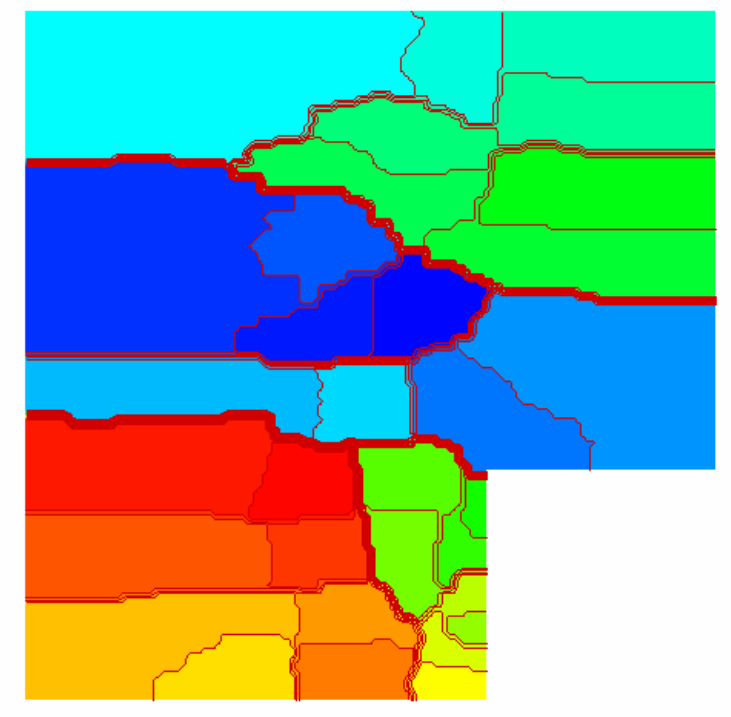

Figure 2. Division of the computational domain showing the load balance.

6

American Institute of Aeronautics and Astronautics 


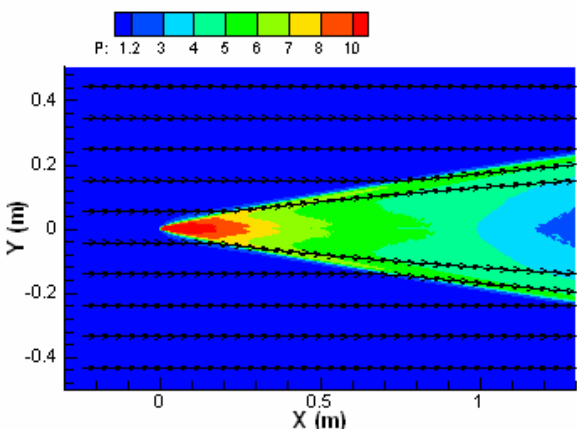

(a) $\mathrm{Kn}=0.002$, angle of attack $=0^{\circ}$

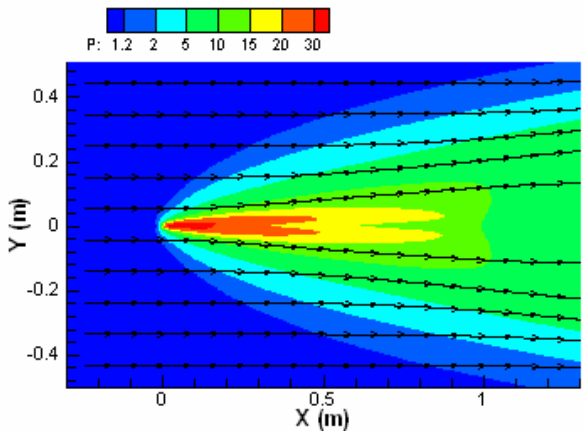

(b) $\mathrm{Kn}=0.02$, angle of attack $=0^{\circ}$

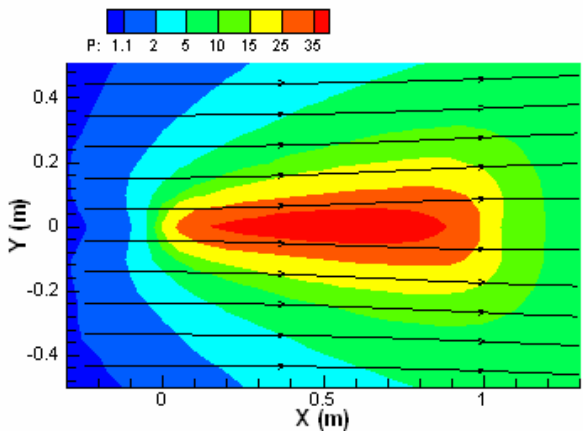

(c) $\mathrm{Kn}=0.2$, angle of attack $=0^{\circ}$

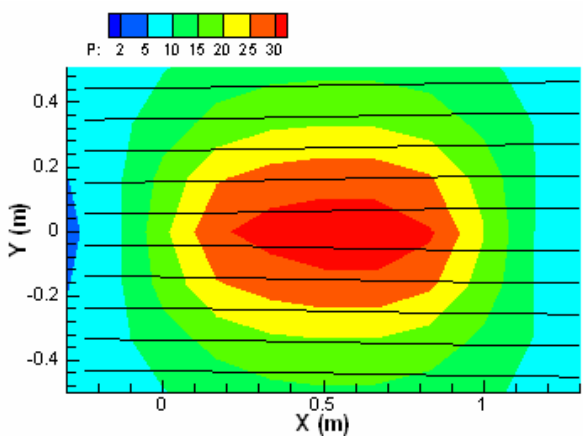

(d) $\mathrm{Kn}=2.0$, angle of attack $=0^{\circ}$

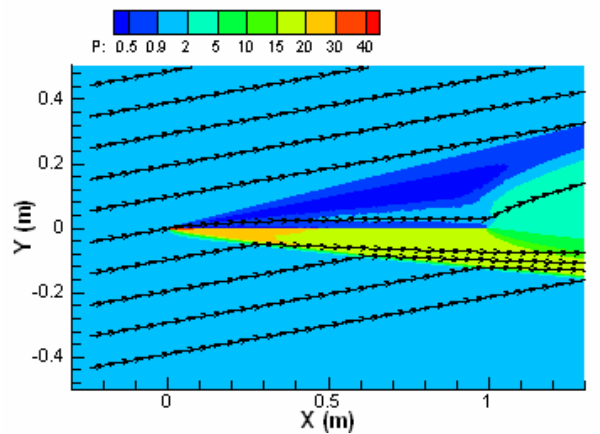

angle of attack $=10^{\circ}$

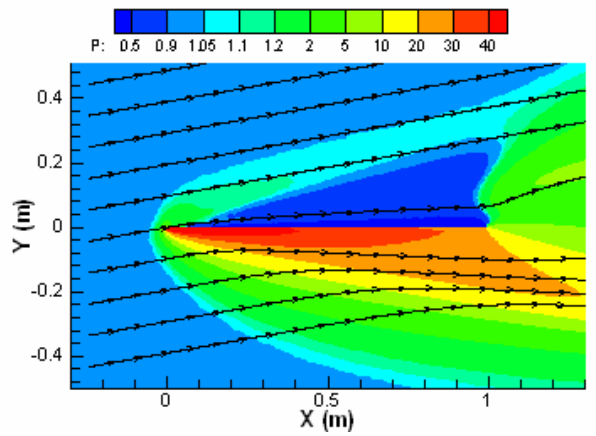

angle of attack $=10^{\circ}$

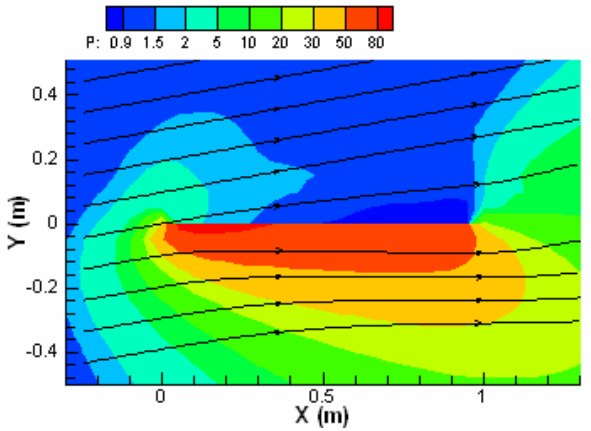

angle of attack $=10^{\circ}$

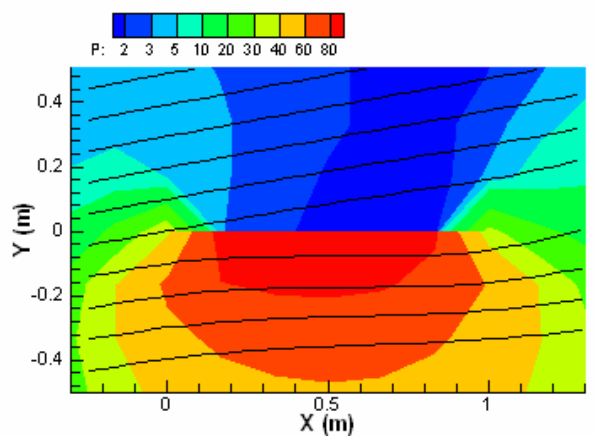

angle of attack $=10^{\circ}$

Figure 3. Knudsen number effects on the pressure field and streamlines. 


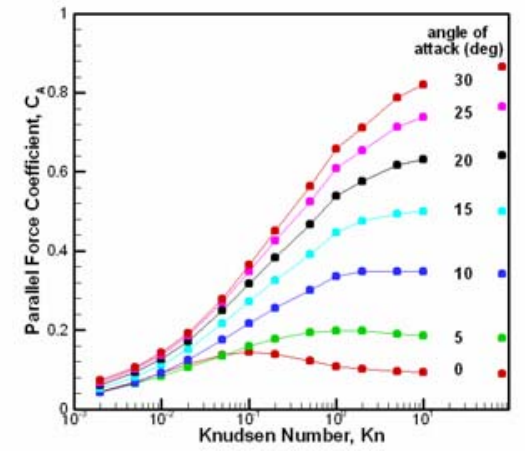

(a) parallel force coefficient

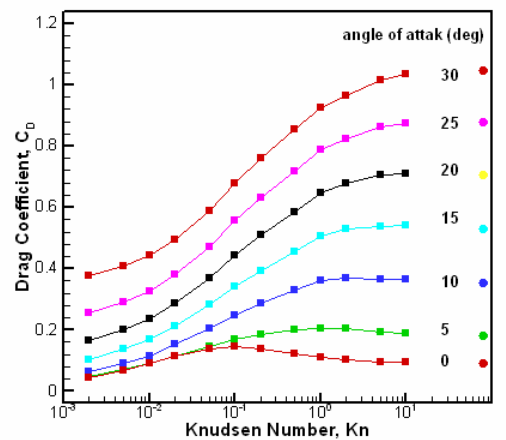

(c) drag coefficient

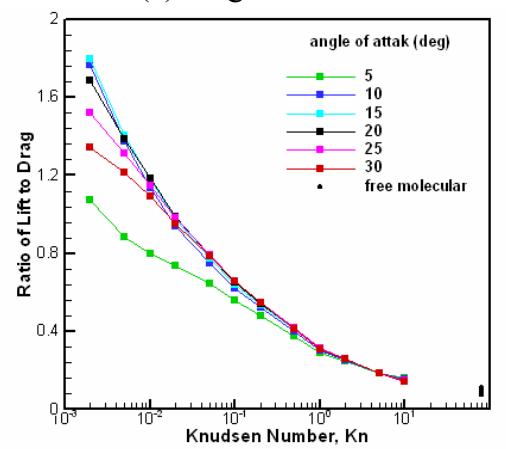

(e) ratio of lift to drag as a function of $\mathrm{Kn}$

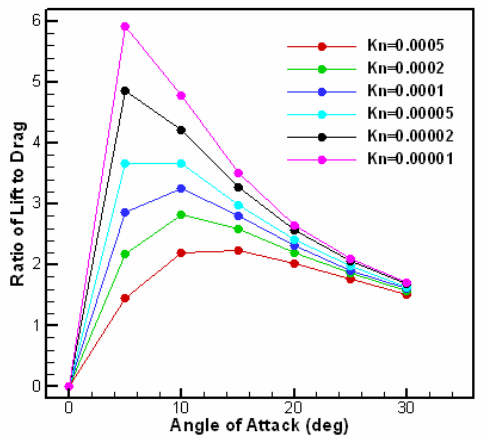

(g) ratio of lift to drag at small Knudsen number (CFD results)

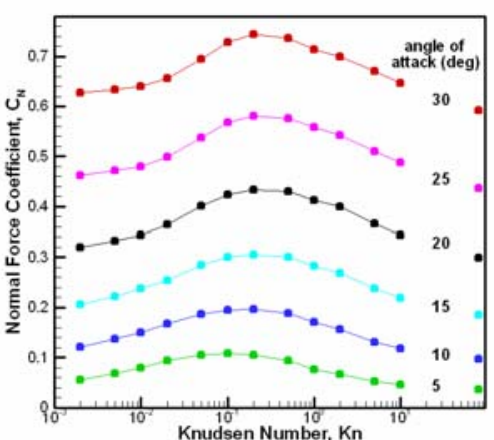

(b) normal force coefficient

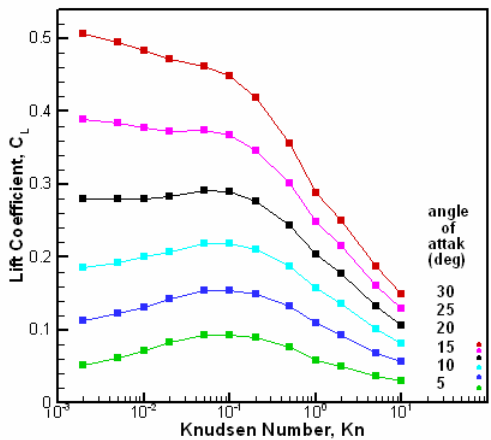

(d) lift coefficient

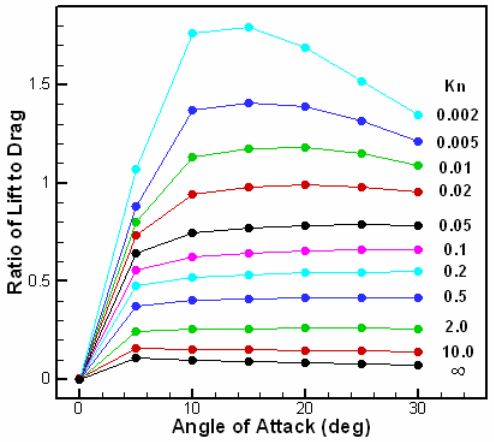

(f) ratio of lift to drag as a function of angle of attack

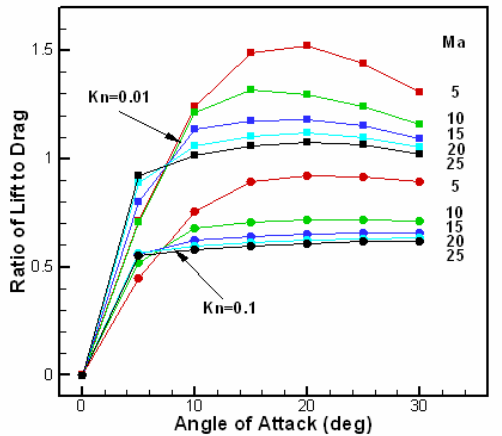

Figure 4. Aerodynamics of hypersonic flat plate (Mach number is 15 except for plot $h$ ). 


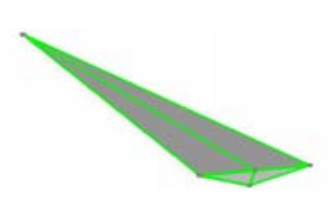

(a) base configuration

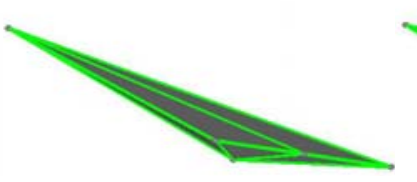

(b) $\mathrm{X}_{4}=0.8_{4 \mathrm{~b}}$

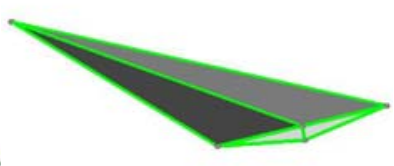

(c) $\mathrm{H}=0.5 \mathrm{H}_{\mathrm{b}}$

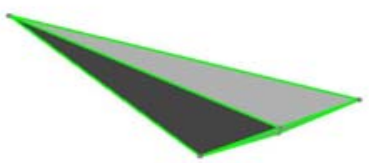

(d) $\mathrm{H}=0.5 \mathrm{H}_{\mathrm{b}}, \mathrm{W}=2 \mathrm{~W}_{\mathrm{b}}$

Figure 5. Delta wing configurations.

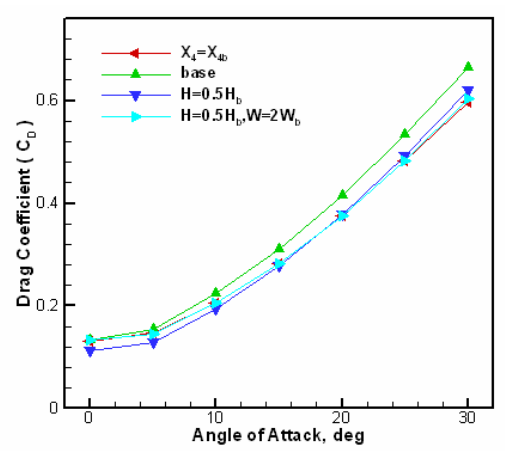

(a) drag coefficient

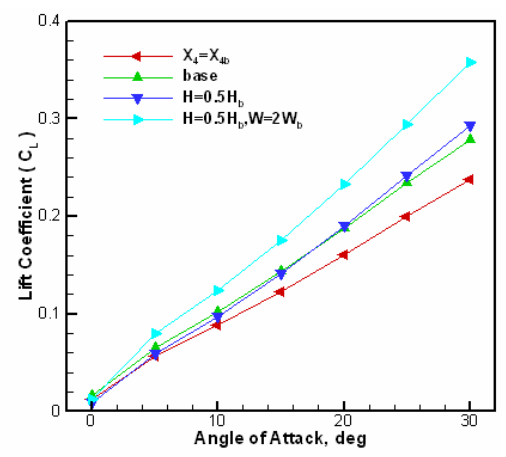

(b) lift coefficient

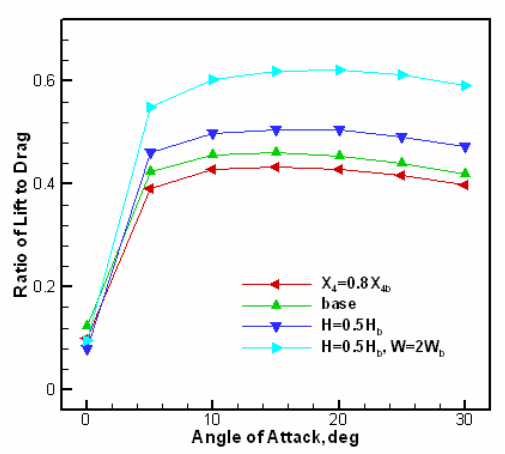

(c) ratio of lift to drag

Figure 6. Aerodynamics of the delta wings when $\mathrm{Ma}=25$ and $\mathrm{Kn}=\mathbf{0 . 0 1 5}$.

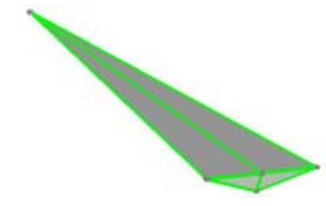

(a) delta wing

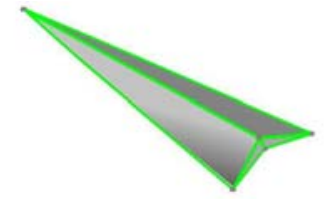

(b) caret waverider

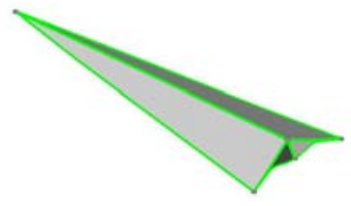

(c) cone-derived waverider

Figure 7. Configuration comparison between delta wing and waveriders.

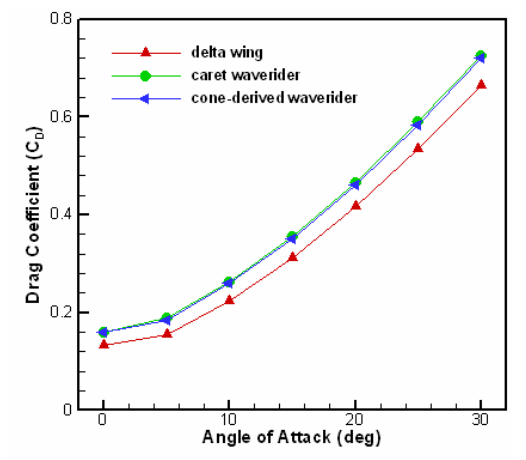

(a) drag coefficient

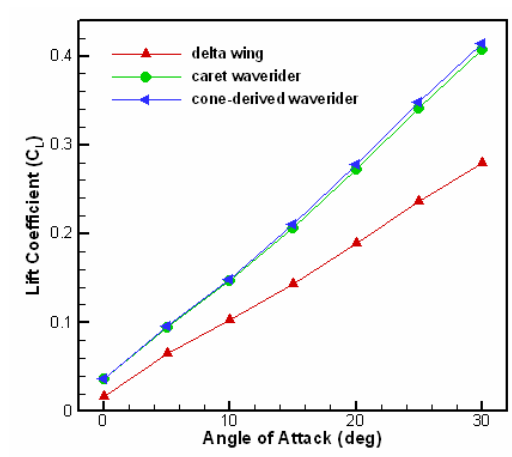

(b) lift coefficient

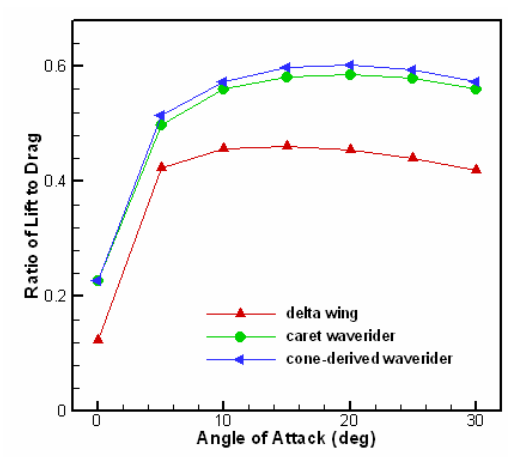

(c) ratio of lift to drag

Figure 8. Comparison of aerodynamics of the delta wing and waveriders when $\mathrm{Ma}=25$ and $\mathrm{Kn}=0.015$. 


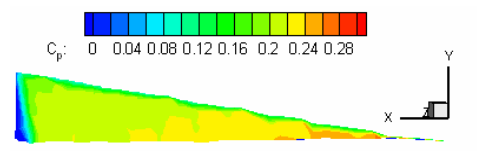

(a) delta wing

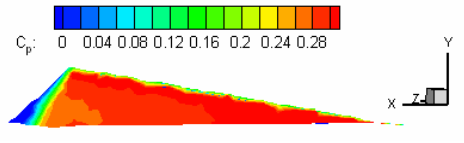

(b) caret waverider

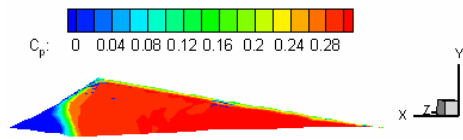

(c) cone-derived waverider

Figure 9. Comparison of surface pressure for the delta wing and waveriders when $\mathrm{Ma}=\mathbf{2 5}$ and $\mathrm{Kn}=\mathbf{0 . 0 1 5}$.

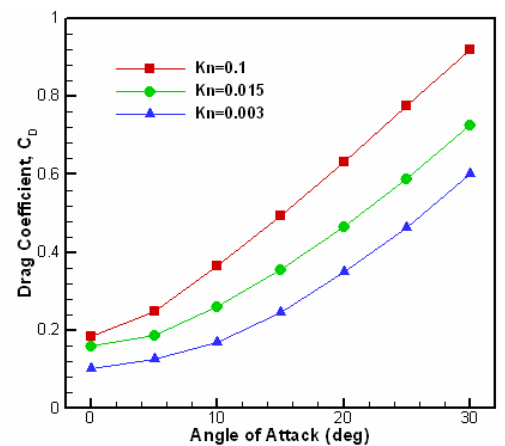

(a) drag coefficient

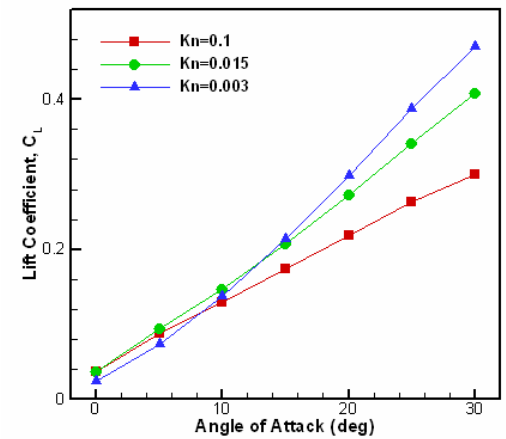

(b) lift coefficient

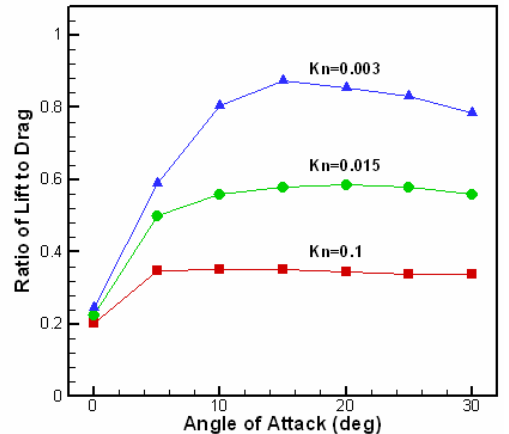

(c) ratio of lift to drag

Figure 10. Aerodynamics of the caret waverider as a function of Knudsen number when Ma=25. 